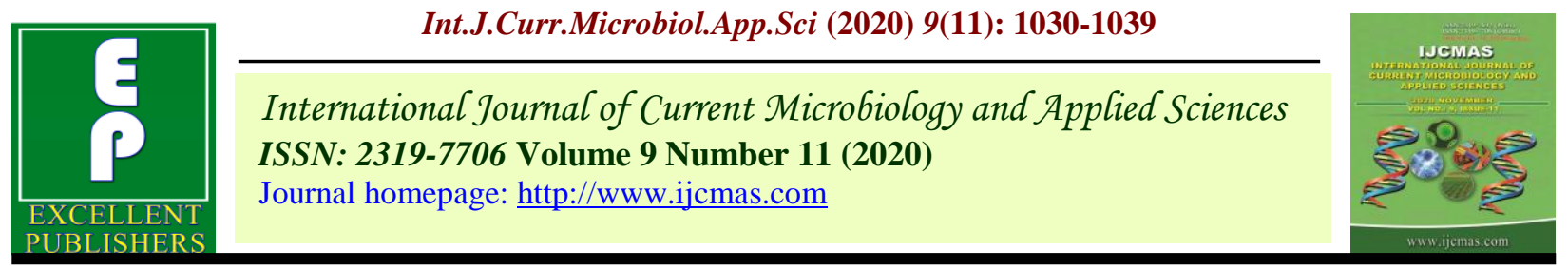

Original Research Article

https://doi.org/10.20546/ijcmas.2020.911.121

\title{
Studies on Genetic Variability, Correlation and Path Coefficient Analysis for Morphological and Yield Traits in Different Arachis spp.
}

\author{
Kalyani Kumari* and N. Sasidharan \\ Department of Seed Science and Technology, Anand Agricultural University, \\ Anand, Gujarat, India \\ *Corresponding author
}

\section{A B S T R A C T}

\begin{tabular}{l} 
Ke y w o r d s \\
$\begin{array}{l}\text { Arachis, Genetic } \\
\text { variability } \\
\text { correlation and Path } \\
\text { coefficient analysis }\end{array}$ \\
\hline Article Info \\
\hline $\begin{array}{l}\text { Accepted: } \\
10 \text { October } 2020 \\
\text { Available Online: } \\
10 \text { November } 2020\end{array}$ \\
\hline
\end{tabular}

\begin{abstract}
Fifty Arachis genotypes belonging to different botanical types viz., spanish bunch, virginia bunch, valencia, peruviana and aequatoriana were evaluated for 28 quantitative characters to study the genetic variability parameters, correlation coefficient and path analysis.. Analysis of variance indicated highly significant differences among genotypes for all the traits. In the present study high magnitude of genetic coefficient of variation (GCV) and phenotypic coefficient of variation (PCV) and high broad sense heritability was observed for 19 characters out of 28 characters. This indicated that these traits were less influenced by the environment and can be improved by simple selection procedure. High genetic advance as percentage of mean was observed for 16 out of 28 characters, which indicate the necessity of utilising these traits for crop improvement for groundnut. From the path analysis study, it was observed that, during kharif season days to maturity had the highest and positive direct effect on kernel yield/plant, while during summer pod yield/plant had the highest and positive direct effect on kernel yield/plant.
\end{abstract}

\section{Introduction}

Groundnut (Arachis hypogaea L.) is one of the important economic crops of the world. It is also called as the "King" of oilseeds or "Wonder nut" and "Poor man's cashewnut". Knowledge of genetic diversity in a crop species is fundamental to its improvement. The characterization of diversity in germplasm collection is important to plant breeders to utilize and to the gene bank curators to manage the collection efficiently and effectively. Assessment of genetic diversity is important steps in the development of molecular breeding programs. Assessment of molecular diversity should facilitate the identification of agronomically valuable and diverse germplasm for use in linkage mapping and genetic enhancement of specific traits in groundnut. Keeping these facts in view, the present investigation variability analysis of quantitative and qualitative characters was undertaken using 50 genotypes of groundnut.

\section{Materials and Methods}

A study was conducted during the kharif season and summer season at Department of Seed Science and Technology, B. A. College of Agriculture, AAU, Anand, in different Arachis spp. The experiment was laid out in 
randomized complete block design with two replications.

The experimental material comprising of Fifty Arachis genotypes belonging to different botanical types viz; spanish bunch, virginia bunch, valencia, peruviana and aequatoriana. Recommended package of practices were followed for raising of the crop. The observations were recorded on five randomly selected competitive plants in each genotype in each replication except days to 50\% flowering and days to emergence which were recorded on plot basis.

The data were subjected to statistical analysis and analysis of variance was calculated (Panse and Sukhatme, 1976) and following genetic parameters were estimated for the character having significant mean square due to genotypes. Phenotypic and genotypic variances were calculated as per the formula given by Johnson et al., (1955). The genotypic coefficient of variation and phenotypic coefficient of variation was estimated as per the formula suggested by Burton (1952). Heritability in broad sense was computed in per cent using the formula suggested by Allard (1960). The extent of genetic advance to be expected from selecting five per cent of superior progeny was computed with the help of the formula given by Allard (1960).

Genotypic correlation coefficient was worked out using the following formula suggested by Hazel et al., (1943). The significance of correlation coefficient was tested against ' $r$ ' value given by Fischer and Yates (1963).

Path coefficient analysis was carried out by using the correlation coefficients to know the direct and indirect effects of these variables on yield as suggested by Wright (1921) and illustrated by Dewey and Lu (1959).

\section{Results and Discussion}

Genotypic and phenotypic correlations reveal the degree of association between different characters and thus aid in selection to improve the yield and yield attributing characters simultaneously. Yield being a complex character is a function of several component characters and their interaction with environment.

Path analysis developed by Wright (1923), is a standardized partial regression analysis for assessment of the magnitude of characters association or correlation of various metric characters with yield and their direct and indirect influence on yield.

In the present study high magnitude of genetic coefficient of variation (GCV) and phenotypic coefficient of variation (PCV)was observed for number of mature pods/plant, number of immature pods/plant, pod yield/plant, kernel yield/plant, number of one seeded pod $\%$, number of three seeded pod\%, hundred pod mass, seed length, seed width hundred seed weight, days to initiation of germination, plant height, length of primary branch, number of secondary branches, number of two seeded pod, pod length, pod width, shelling\% and SMK\%.

It indicated that higher the amount of genetic component of variation in these characters, greater the scope for its improvement through selection. High GCV values for these characters were also observed by Yusuf et al., (2017).

High broad sense heritability estimates were recorded for most of traits viz., days to maturity, plant height, length of primary branch, leaf length, number of mature pods/plant, number of immature pods/plant, pod yield/plant, kernel yield/plant, number of one seeded pods $\%$, number of two seeded 
pods $\%$, number of three seeded pods $\%$, pod length, pod width, hundred pod mass, shelling $\%$, seed length, seed width, hundred seed weight and SMK\%indicating that these traits were less influenced by the environment.
These traits could be improved by simple selection procedure. Similar results were also observed by Zaman et al., (2011) and Patil et al., (2015), Gupta et al., (2015) and Chavadhari et al., (2017) (Table 1-6).

Table.1 The estimates of genotypic $\left(\sigma_{\mathrm{g}}{ }^{2}\right)$ and phenotypic $\left(\sigma_{\mathrm{p}}{ }^{2}\right)$ variance and other genetic parameters for different characters in groundnut during kharif season

\begin{tabular}{|c|c|c|c|c|c|c|c|c|}
\hline S.n. & Character & $\sigma_{\mathrm{g}}^{2}$ & $\sigma_{p}^{2}$ & $\begin{array}{l}\text { GCV } \\
(\%)\end{array}$ & $\begin{array}{l}\text { PCV } \\
(\%)\end{array}$ & $\begin{array}{l}\mathbf{H}^{2} \\
(\%)\end{array}$ & $\mathbf{R}$ & $\begin{array}{c}\text { GA\% over } \\
\text { mean }\end{array}$ \\
\hline 1. & Days to initiation of germination & 0.67 & 1.45 & 13.68 & 20.06 & 47 & 1.15 & 19.17 \\
\hline 2. & Days to initiation of flowering & 0.94 & 2.07 & 4.04 & 6.00 & 45 & 1.34 & 5.59 \\
\hline 3. & Days to $50 \%$ flowering & 1.21 & 5.16 & 3.71 & 7.66 & 24 & 1.10 & 3.71 \\
\hline 4. & Days to maturity & 61.43 & 74.65 & 6.52 & 7.19 & 82 & 14.65 & 12.18 \\
\hline 5. & Plant height $(\mathrm{cm})$ & 33.17 & 48.34 & 18.54 & 22.38 & 69 & 9.83 & 31.64 \\
\hline 6. & Length of primary branch $(\mathrm{cm})$ & 14.29 & 19.48 & 11.38 & 13.28 & 73 & 6.67 & 29.59 \\
\hline 7. & Number of primary branches & 0.32 & 1.31 & 7.60 & 15.33 & 25 & 0.58 & 89.41 \\
\hline 8. & Number of secondary branches & 0.79 & 1.58 & 15.00 & 21.18 & 50 & 1.30 & 9.78 \\
\hline 9. & Leaf length $(\mathrm{cm})$ & 0.29 & 0.45 & 9.33 & 11.59 & 65 & 0.90 & 15.52 \\
\hline 10. & Leaf width (cm) & 0.04 & 0.09 & 6.53 & 10.01 & 43 & 0.26 & 8.81 \\
\hline 11. & Leaf length/leaf width & 0.02 & 0.09 & 7.24 & 14.16 & 26 & 0.16 & 7.66 \\
\hline 12. & Number of mature pods / plant & 135.85 & 138.73 & 35.75 & 36.13 & 98 & 23.76 & 96.98 \\
\hline 13. & Number of immature pods / plant & 3.39 & 3.96 & 39.01 & 42.16 & 86 & 3.51 & 74.36 \\
\hline 14. & Pod yield/plant (g) & 102.05 & 105.91 & 33.15 & 33.77 & 96 & 20.43 & 67.03 \\
\hline 15. & Kernel yield/plant (g) & 57.97 & 62.80 & 35.52 & 36.97 & 92 & 15.07 & 70.32 \\
\hline 16. & Number of one seeded pod (\%) & 104.27 & 108.40 & 79.40 & 80.96 & 96 & 20.63 & 160.42 \\
\hline 17. & Number of two seeded pod (\%) & 112.03 & 116.69 & 12.54 & 12.80 & 96 & 21.36 & 25.31 \\
\hline 18. & Number of three seeded pod (\%) & 0.75 & 0.78 & 50.10 & 51.25 & 96 & 1.74 & 70.16 \\
\hline 19. & Pod length $(\mathrm{cm})$ & 0.19 & 0.21 & 17.77 & 18.55 & 92 & 0.86 & 35.10 \\
\hline 20. & Pod width $(\mathrm{cm})$ & 0.03 & 0.03 & 12.68 & 13.39 & 90 & 0.32 & 25.20 \\
\hline 21. & Hundred pod mass (g) & 485.50 & 495.95 & 22.44 & 22.68 & 98 & 44.91 & 45.73 \\
\hline 22. & Shelling per cent (S \%) & 57.82 & 63.60 & 11.41 & 11.97 & 91 & 14.94 & 22.42 \\
\hline 23. & Seed length $(\mathrm{cm})$ & 0.11 & 0.12 & 24.30 & 24.74 & 97 & 0.67 & 48.91 \\
\hline 24. & Seed width (cm) & 0.06 & 0.07 & 28.72 & 30.73 & 87 & 0.48 & 104.35 \\
\hline 25. & Hundred seed weight (g) & 153.38 & 156.51 & 26.22 & 26.49 & 98 & 25.26 & 53.48 \\
\hline 26. & Sound mature kernel (SMK \%) & 98.03 & 105.34 & 11.15 & 11.56 & 93 & 19.68 & 22.16 \\
\hline 27. & Oil content $(\%)$ & 2.59 & 4.30 & 3.42 & 4.41 & 60 & 2.57 & 5.47 \\
\hline 28. & Protein content (\%) & 0.43 & 1.80 & 2.26 & 4.65 & 24 & 0.65 & 2.26 \\
\hline
\end{tabular}

Genetic Advance Values $(\mathrm{K}=2.06), \mathrm{R}=$ Genetic gain 
Table. 2 The estimates of genotypic $\left(\sigma_{\mathrm{g}}{ }^{2}\right)$ and phenotypic $\left(\sigma_{\mathrm{p}}{ }^{2}\right)$ variance and other genetic parameters for different characters in groundnut during summer season

\begin{tabular}{|c|c|c|c|c|c|c|c|c|}
\hline S.No. & Character & $\sigma_{\mathrm{g}}^{2}$ & $\sigma_{p}^{2}$ & $\begin{array}{l}\text { GCV } \\
(\%)\end{array}$ & $\begin{array}{l}\text { PCV } \\
(\%)\end{array}$ & $\begin{array}{c}\mathbf{H}^{2} \\
(\%)\end{array}$ & $\mathbf{R}$ & $\begin{array}{l}\text { GA\% over } \\
\text { mean }\end{array}$ \\
\hline 1. & $\begin{array}{l}\text { Days to initiation of } \\
\text { germination }\end{array}$ & 1.23 & 2.10 & 17.26 & 22.58 & 58 & 1.74 & 27.15 \\
\hline 2. & Days to initiation of flowering & 3.24 & 5.38 & 6.84 & 8.82 & 60 & 2.88 & 10.95 \\
\hline 3. & Days to $50 \%$ flowering & 1.32 & 5.78 & 3.59 & 7.51 & 23 & 1.13 & 3.53 \\
\hline 4. & Days to maturity & 77.14 & 89.02 & 7.97 & 8.56 & 87 & 16.84 & 15.28 \\
\hline 5. & Plant height $(\mathrm{cm})$ & 42.77 & 50.68 & 23.48 & 25.56 & 84 & 12.38 & 44.45 \\
\hline 6. & $\begin{array}{l}\text { Length of primary branch } \\
(\mathrm{cm})\end{array}$ & 20.91 & 25.42 & 15.07 & 16.62 & 82 & 8.54 & 28.15 \\
\hline 7. & Number of primary branches & 1.22 & 2.01 & 19.08 & 24.46 & 61 & 1.78 & 30.74 \\
\hline 8. & $\begin{array}{l}\text { Number of secondary } \\
\text { branches }\end{array}$ & 0.72 & 1.11 & 20.34 & 25.20 & 65 & 1.41 & 33.73 \\
\hline 9. & Leaf length $(\mathrm{cm})$ & 0.41 & 0.57 & 11.98 & 14.16 & 72 & 1.11 & 20.86 \\
\hline 10. & Leaf width $(\mathrm{cm})$ & 0.08 & 0.12 & 10.29 & 12.75 & 65 & 0.46 & 17.10 \\
\hline 11. & Leaf length/leaf width & 0.04 & 0.09 & 10.44 & 15.30 & 47 & 0.29 & 14.50 \\
\hline 12. & $\begin{array}{l}\text { Number of mature pods / } \\
\text { plants }\end{array}$ & 137.32 & 140.60 & 38.55 & 39.00 & 98 & 23.86 & 78.49 \\
\hline 13. & $\begin{array}{l}\text { Number of immature pods / } \\
\text { plants }\end{array}$ & 3.93 & 5.13 & 28.73 & 32.83 & 77 & 3.57 & 51.74 \\
\hline 14. & Pod yield/plant (g) & 93.58 & 101.23 & 34.98 & 36.38 & 92 & 19.16 & 69.27 \\
\hline 15. & Kernel yield/plant (g) & 52.54 & 56.89 & 38.71 & 40.28 & 92 & 14.35 & 76.66 \\
\hline 16. & $\begin{array}{l}\text { Number of one seeded pod } \\
(\%)\end{array}$ & 111.34 & 116.17 & 68.16 & 69.63 & 96 & 21.28 & 137.65 \\
\hline 17. & $\begin{array}{l}\text { Number of two seeded pod } \\
(\%)\end{array}$ & 117.03 & 121.88 & 13.19 & 13.47 & 96 & 21.84 & 26.60 \\
\hline 18. & $\begin{array}{l}\text { Number of three seeded pod } \\
(\%)\end{array}$ & 0.67 & 0.70 & 49.86 & 50.82 & 96 & 1.65 & 75.34 \\
\hline 19. & Pod length $(\mathrm{cm})$ & 0.18 & 0.19 & 18.34 & 18.80 & 95 & 0.85 & 71.43 \\
\hline 20. & Pod width $(\mathrm{cm})$ & 0.03 & 0.03 & 13.41 & 14.15 & 90 & 0.32 & 26.45 \\
\hline 21. & Hundred pod mass (g) & 488.45 & 496.84 & 23.47 & 23.67 & 98 & 45.14 & 47.93 \\
\hline 22. & Shelling per cent (S \%) & 62.29 & 67.95 & 12.20 & 12.74 & 92 & 15.57 & 24.07 \\
\hline 23. & Seed length $(\mathrm{cm})$ & 0.12 & 0.13 & 26.90 & 27.66 & 95 & 0.70 & 53.85 \\
\hline 24. & Seed width $(\mathrm{cm})$ & 0.05 & 0.06 & 28.23 & 29.83 & 90 & 0.44 & 55.00 \\
\hline 25. & Hundred seed weight ( $g$ ) & 155.43 & 158.84 & 27.32 & 27.62 & 98 & 25.49 & 55.69 \\
\hline 26. & $\begin{array}{l}\text { Sound mature kernel (SMK } \\
\% \text { ) }\end{array}$ & 99.44 & 107.14 & 11.48 & 11.92 & 93 & 19.79 & 22.79 \\
\hline 27. & Oil content $(\%)$ & 3.06 & 5.33 & 3.88 & 5.11 & 57 & 2.73 & 6.05 \\
\hline 28. & Protein content (\%) & 0.49 & 2.03 & 2.52 & 5.12 & 24 & 0.71 & 2.55 \\
\hline
\end{tabular}


Table.3 Genotypic and phenotypic correlations between kernel yield and other component characters in groundnut during kharif season

\begin{tabular}{|c|c|c|c|c|c|c|c|c|c|c|c|c|c|c|c|}
\hline \multirow[t]{2}{*}{ S.No. } & \multirow[t]{2}{*}{ Character } & & 1 & 2 & 3 & 4 & 5 & 6 & 7 & 8 & 9 & 10 & 11 & 12 & 13 \\
\hline & & & $\begin{array}{l}\text { Kernel } \\
\text { yield/pl } \\
\text { ant }(g)\end{array}$ & $\begin{array}{l}\text { Days to } \\
50 \% \\
\text { flowerin } \\
\mathrm{g}\end{array}$ & $\begin{array}{l}\text { Days to } \\
\text { maturity }\end{array}$ & $\begin{array}{l}\text { Plant } \\
\text { height } \\
(\mathrm{cm})\end{array}$ & $\begin{array}{c}\text { Number of } \\
\text { primary } \\
\text { branches }\end{array}$ & $\begin{array}{l}\text { Number } \\
\text { of mature } \\
\text { pods / } \\
\text { plant }\end{array}$ & $\begin{array}{c}\text { Pod } \\
\text { yield/plant } \\
(\mathrm{g})\end{array}$ & $\begin{array}{l}\text { Hundred } \\
\text { pod mass } \\
\quad(\mathrm{g})\end{array}$ & $\begin{array}{c}\text { Shelling } \\
\text { per cent (S } \\
\%)\end{array}$ & $\begin{array}{c}\text { Hundred } \\
\text { seed weight } \\
\text { (g) }\end{array}$ & $\begin{array}{c}\text { Sound } \\
\text { mature } \\
\text { kernel } \\
(\text { SMK \%) }\end{array}$ & $\begin{array}{l}\text { Oil content } \\
(\%)\end{array}$ & $\begin{array}{c}\text { Protein } \\
\text { content }(\%)\end{array}$ \\
\hline \multirow[t]{2}{*}{1.} & \multirow[t]{2}{*}{$\begin{array}{l}\text { Kernel } \\
\text { yield/plant (g) }\end{array}$} & $r_{g}$ & 1.000 & -0.020 & 0.087 & - $0.356^{*}$ & 0.027 & $0.798 * *$ & $0.986 * *$ & $0.539 * *$ & -0.158 & 0.268 & -0.097 & -0.250 & 0.013 \\
\hline & & $r_{p}$ & 1.000 & 0.015 & 0.080 & -0.266 & -0.070 & $0.749 * *$ & $0.942 * *$ & $0.522 * *$ & -0.160 & 0.246 & -0.099 & -0.227 & 0.025 \\
\hline \multirow[t]{2}{*}{2.} & \multirow{2}{*}{$\begin{array}{l}\text { Days to } 50 \% \\
\text { flowering }\end{array}$} & $r_{g}$ & & 1.000 & $0.949 * *$ & -0.093 & $-0.794 * *$ & -0.250 & 0.083 & -0.046 & 0.029 & $0.365 * *$ & -0.059 & $0.294 *$ & 0.207 \\
\hline & & $r_{p}$ & & 1.000 & $0.275^{*}$ & -0.080 & -0.068 & -0.120 & 0.000 & -0.012 & 0.038 & 0.180 & -0.027 & 0.127 & -0.037 \\
\hline \multirow[t]{2}{*}{3.} & \multirow[t]{2}{*}{ Days to maturity } & $r_{g}$ & & & 1.000 & $0.307 *$ & $-0.396 * *$ & 0.049 & 0.091 & $0.309 *$ & -0.142 & 0.098 & -0.251 & -0.100 & 0.275 \\
\hline & & $r_{p}$ & & & 1.000 & 0.255 & -0.250 & 0.035 & 0.087 & $0.280^{*}$ & -0.116 & 0.077 & -0.225 & -0.034 & 0.147 \\
\hline \multirow[t]{2}{*}{4.} & \multirow[t]{2}{*}{ Plant height $(\mathrm{cm})$} & $r_{g}$ & & & & 1.000 & -0.059 & $-0.291 *$ & -0.347 & -0.040 & 0.133 & -0.018 & -0.103 & 0.134 & $0.314^{*}$ \\
\hline & & $r_{p}$ & & & & 1.000 & -0.035 & -0.244 & -0.267 & -0.043 & 0.136 & -0.023 & -0.083 & 0.043 & 0.119 \\
\hline \multirow[t]{2}{*}{5.} & \multirow{2}{*}{$\begin{array}{l}\text { Number of } \\
\text { primary branches }\end{array}$} & $r_{g}$ & & & & & 1.000 & -0.005 & -0.037 & -0.186 & -0.381 & $0.346^{*}$ & 0.264 & -0.318 & $0.716^{* *}$ \\
\hline & & $r_{p}$ & & & & & 1.000 & 0.023 & 0.008 & -0.130 & -0.157 & 0.167 & 0.131 & 0.081 & 0.002 \\
\hline \multirow[t]{2}{*}{6.} & \multirow{2}{*}{$\begin{array}{l}\text { Number of } \\
\text { mature pods / } \\
\text { plant }\end{array}$} & $r_{g}$ & & & & & & 1.000 & $0.783^{* *}$ & $0.281 *$ & -0.072 & -0.045 & -0.028 & $-0.513 * *$ & 0.242 \\
\hline & & $r_{p}$ & & & & & & 1.000 & $0.762 * *$ & $0.274 *$ & -0.065 & -0.046 & -0.026 & $-0.386^{* *}$ & 0.135 \\
\hline \multirow[t]{2}{*}{7.} & \multirow{2}{*}{$\begin{array}{l}\text { Pod yield/plant } \\
\text { (g) }\end{array}$} & $r_{g}$ & & & & & & & 1.000 & $0.499 * *$ & -0.055 & $0.336^{*}$ & -0.084 & -0.204 & 0.025 \\
\hline & & $r_{p}$ & & & & & & & 1.000 & $0.480 * *$ & -0.041 & $0.323 *$ & -0.077 & -0.151 & -0.015 \\
\hline \multirow[t]{2}{*}{8.} & \multirow{2}{*}{$\begin{array}{l}\text { Hundred pod } \\
\text { mass }(\mathrm{g})\end{array}$} & $r_{g}$ & & & & & & & & 1.000 & $-0.483^{* *}$ & $0.427 * *$ & $-0.498 * *$ & -0.183 & 0.100 \\
\hline & & $r_{p}$ & & & & & & & & 1.000 & $-0.446 * *$ & $0.413 * *$ & $-0.467 * *$ & -0.142 & 0.064 \\
\hline \multirow[t]{2}{*}{9.} & \multirow{2}{*}{$\begin{array}{l}\text { Shelling per cent } \\
(\mathrm{S} \%)\end{array}$} & $r_{g}$ & & & & & & & & & 1.000 & 0.187 & $0.389 * *$ & $0.454 * *$ & $-0.664 * *$ \\
\hline & & $r_{p}$ & & & & & & & & & 1.000 & 0.166 & $0.376 * *$ & $0.348 *$ & $-0.289 *$ \\
\hline \multirow[t]{2}{*}{10.} & \multirow{2}{*}{$\begin{array}{l}\text { Hundred seed } \\
\text { weight }(\mathrm{g})\end{array}$} & $r_{g}$ & & & & & & & & & & 1.000 & -0.168 & $0.484 * *$ & $-0.497 * *$ \\
\hline & & $r_{p}$ & & & & & & & & & & 1.000 & -0.165 & $0.346^{*}$ & -0.252 \\
\hline \multirow[t]{2}{*}{11.} & \multirow{2}{*}{$\begin{array}{l}\text { Sound mature } \\
\text { kernel (SMK \%) }\end{array}$} & $r_{g}$ & & & & & & & & & & & 1.000 & 0.242 & $-0.348^{*}$ \\
\hline & & $r_{p}$ & & & & & & & & & & & 1.000 & 0.156 & -0.162 \\
\hline \multirow[t]{2}{*}{12.} & \multirow[t]{2}{*}{ Oil content $(\%)$} & $r_{g}$ & & & & & & & & & & & & 1.000 & $-0.492 * *$ \\
\hline & & $r_{p}$ & & & & & & & & & & & & 1.000 & -0.244 \\
\hline \multirow[t]{2}{*}{13.} & Protein content & $r_{g}$ & & & & & & & & & & & & & 1.000 \\
\hline & & $r_{p}$ & & & & & & & & & & & & & 1.000 \\
\hline
\end{tabular}

$*$,** -Significant at $5 \%$ and $1 \%$ level of significance, respectively 
Table.4 Genotypic and phenotypic correlations between kernel yield and other component characters in groundnut during summer season

\begin{tabular}{|c|c|c|c|c|c|c|c|c|c|c|c|c|c|c|c|}
\hline \multirow[t]{2}{*}{ S.No. } & \multirow[t]{2}{*}{ Character } & & 1 & 2 & 3 & 4 & 5 & 6 & 7 & 8 & 9 & 10 & 11 & 12 & 13 \\
\hline & & & $\begin{array}{l}\text { Kernel } \\
\text { yield/plant } \\
(\mathrm{g})\end{array}$ & $\begin{array}{c}\text { Days to } \\
50 \% \\
\text { flowering }\end{array}$ & $\begin{array}{l}\text { Days to } \\
\text { maturity }\end{array}$ & $\begin{array}{l}\text { Plant } \\
\text { height } \\
(\mathrm{cm})\end{array}$ & $\begin{array}{l}\text { Number of } \\
\text { primary } \\
\text { branches }\end{array}$ & $\begin{array}{l}\text { Number of } \\
\text { mature pods } \\
\text { / plant }\end{array}$ & $\begin{array}{c}\text { Pod } \\
\text { yield/plant } \\
(\mathrm{g})\end{array}$ & $\begin{array}{l}\text { Hundred } \\
\text { pod mass } \\
(\mathrm{g})\end{array}$ & $\begin{array}{l}\text { Shelling per } \\
\text { cent }(\mathrm{S} \%)\end{array}$ & $\begin{array}{l}\text { Hundred } \\
\text { seed weight } \\
\text { (g) }\end{array}$ & $\begin{array}{c}\text { Sound } \\
\text { mature } \\
\text { kernel } \\
(\text { SMK \%) }\end{array}$ & $\begin{array}{l}\text { Oil content } \\
\quad(\%)\end{array}$ & $\begin{array}{c}\text { Protein } \\
\text { content }(\%)\end{array}$ \\
\hline \multirow[t]{2}{*}{1.} & \multirow{2}{*}{$\begin{array}{l}\text { Kernel yield/plant } \\
\text { (g) }\end{array}$} & $r_{g}$ & 1.000 & 0.189 & 0.077 & -0.278 & -0.057 & $0.796 * *$ & $0.993 * *$ & $0.533 * *$ & -0.199 & 0.243 & -0.088 & $-0.302 *$ & 0.058 \\
\hline & & $r_{p}$ & 1.000 & 0.110 & 0.055 & -0.245 & -0.092 & $0.746^{* *}$ & $0.939 * *$ & $0.511 * *$ & -0.164 & 0.232 & -0.081 & -0.197 & 0.032 \\
\hline \multirow[t]{2}{*}{2.} & \multirow{2}{*}{$\begin{array}{l}\text { Days to } 50 \% \\
\text { flowering }\end{array}$} & $r_{g}$ & & 1.000 & $0.805 * *$ & -0.222 & $-0.519 * *$ & -0.111 & 0.245 & 0.146 & -0.094 & 0.275 & 0.067 & $0.320^{*}$ & 0.152 \\
\hline & & $r_{p}$ & & 1.000 & $0.326^{*}$ & -0.048 & -0.147 & -0.032 & 0.107 & 0.073 & 0.005 & 0.129 & 0.029 & 0.229 & -0.117 \\
\hline \multirow[t]{2}{*}{3.} & \multirow[t]{2}{*}{ Days to maturity } & $r_{g}$ & & & 1.000 & $0.332 *$ & -0.209 & -0.018 & 0.078 & $0.292 *$ & -0.222 & 0.038 & -0.260 & -0.054 & $0.428 * *$ \\
\hline & & $r_{p}$ & & & 1.000 & $0.306^{*}$ & -0.133 & -0.009 & 0.064 & $0.267 *$ & -0.205 & 0.044 & -0.250 & -0.074 & 0.164 \\
\hline \multirow[t]{2}{*}{4.} & \multirow[t]{2}{*}{ Plant height $(\mathrm{cm})$} & $r_{g}$ & & & & 1.000 & -0.100 & $-0.277 *$ & -0.249 & -0.012 & 0.161 & 0.066 & -0.123 & 0.103 & $0.306^{*}$ \\
\hline & & $r_{p}$ & & & & 1.000 & -0.063 & -0.252 & -0.225 & -0.015 & 0.146 & 0.059 & -0.139 & 0.101 & 0.183 \\
\hline \multirow[t]{2}{*}{5.} & \multirow{2}{*}{$\begin{array}{l}\text { Number of primary } \\
\text { branches }\end{array}$} & $r_{g}$ & & & & & 1.000 & -0.156 & -0.102 & -0.131 & $-0.373 * *$ & -0.195 & 0.045 & -0.224 & $0.374 * *$ \\
\hline & & $r_{p}$ & & & & & 1.000 & -0.108 & -0.089 & -0.105 & -0.261 & -0.177 & 0.040 & -0.120 & 0.070 \\
\hline \multirow[t]{2}{*}{6.} & \multirow{2}{*}{$\begin{array}{l}\text { Number of mature } \\
\text { pods / plant }\end{array}$} & $r_{g}$ & & & & & & 1.000 & $0.780 * *$ & $0.279 *$ & $0.279 * *$ & -0.055 & -0.032 & $-0.509 * *$ & $0.289 *$ \\
\hline & & $r_{p}$ & & & & & & 1.000 & $0.759 * *$ & 0.270 & -0.062 & -0.054 & -0.032 & $-0.372 * *$ & 0.156 \\
\hline \multirow[t]{2}{*}{7.} & \multirow[t]{2}{*}{ Pod yield/plant (g) } & $r_{g}$ & & & & & & & 1.000 & $0.504 * *$ & -0.051 & $0.332 *$ & -0.093 & -0.190 & 0.067 \\
\hline & & $\mathrm{r}_{\mathrm{p}}$ & & & & & & & 1.000 & $0.483 * *$ & -0.038 & $0.324 *$ & -0.084 & -0.140 & 0.019 \\
\hline \multirow[t]{2}{*}{8.} & \multirow{2}{*}{$\begin{array}{l}\text { Hundred pod mass } \\
(\mathrm{g})\end{array}$} & $r_{g}$ & & & & & & & & 1.000 & $-0.476 * *$ & $0.415^{* *}$ & $-0.494 * *$ & -0.177 & 0.133 \\
\hline & & $r_{p}$ & & & & & & & & 1.000 & $-0.456 * *$ & $0.407 * *$ & $-0.470 * *$ & -0.124 & 0.070 \\
\hline \multirow[t]{2}{*}{9.} & \multirow{2}{*}{$\begin{array}{l}\text { Shelling per cent } \\
(\mathrm{S} \%)\end{array}$} & $r_{g}$ & & & & & & & & & 1.000 & 0.223 & $0.387 * *$ & $0.444 * *$ & $-0.582 * *$ \\
\hline & & $\mathrm{r}_{\mathrm{p}}$ & & & & & & & & & 1.000 & 0.199 & $0.364 * *$ & $0.342 *$ & -0.244 \\
\hline \multirow[t]{2}{*}{10.} & \multirow{2}{*}{$\begin{array}{l}\text { Hundred seed } \\
\text { weight }(\mathrm{g})\end{array}$} & $r_{g}$ & & & & & & & & & & 1.000 & -0.174 & $0.503 * *$ & $-0.494 * *$ \\
\hline & & $r_{p}$ & & & & & & & & & & 1.000 & -0.162 & $0.355 * *$ & -0.237 \\
\hline \multirow[t]{2}{*}{11.} & \multirow{2}{*}{$\begin{array}{l}\text { Sound mature } \\
\text { kernel (SMK \%) }\end{array}$} & $r_{g}$ & & & & & & & & & & & 1.000 & 0.177 & -0.275 \\
\hline & & $r_{p}$ & & & & & & & & & & & 1.000 & 0.118 & -0.130 \\
\hline \multirow[t]{2}{*}{12.} & \multirow[t]{2}{*}{ Oil content (\%) } & $r_{g}$ & & & & & & & & & & & & 1.000 & $-0.451 * *$ \\
\hline & & $r_{p}$ & & & & & & & & & & & & 1.000 & -0.223 \\
\hline \multirow[t]{2}{*}{13.} & Protein content & $r_{g}$ & & & & & & & & & & & & & 1.000 \\
\hline & $(\%)$ & $r_{p}$ & & & & & & & & & & & & & 1.000 \\
\hline
\end{tabular}

$*$, ** -Significant at $5 \%$ and $1 \%$ level of significance, respectively 
Table.5 Genotypic path coefficient analysis showing direct (Diagonal) and indirect effects of different characters on kernel yield in groundnut during kharif season

\begin{tabular}{|c|c|c|c|c|c|c|c|c|c|c|c|c|c|}
\hline Characters & $\begin{array}{c}\text { Days to } 50 \\
\% \\
\text { flowering }\end{array}$ & $\begin{array}{c}\text { Days to } \\
\text { maturity }\end{array}$ & $\begin{array}{c}\text { Plant } \\
\text { height } \\
\text { (cm) }\end{array}$ & $\begin{array}{l}\text { Number } \\
\text { of } \\
\text { primary } \\
\text { branches }\end{array}$ & $\begin{array}{l}\text { Number } \\
\text { of } \\
\text { mature } \\
\text { pods / } \\
\text { plant }\end{array}$ & $\begin{array}{c}\text { Pod } \\
\text { yield/pla } \\
\text { nt (g) }\end{array}$ & $\begin{array}{l}\text { Hundre } \\
\text { d pod } \\
\operatorname{mass}(g)\end{array}$ & $\begin{array}{c}\text { Shelling } \\
\text { per cent } \\
\text { (S \%) }\end{array}$ & $\begin{array}{l}\text { Hundred } \\
\text { seed } \\
\text { weight }(g)\end{array}$ & $\begin{array}{c}\text { Sound } \\
\text { mature } \\
\text { kernel } \\
(\text { SMK \%) }\end{array}$ & $\begin{array}{c}\text { Oil content } \\
(\%)\end{array}$ & $\begin{array}{l}\text { Protein } \\
\text { content } \\
(\%)\end{array}$ & $\begin{array}{c}\text { Genotypic } \\
\text { correlation } \\
\text { with Kernel } \\
\text { yield/plant (g) }\end{array}$ \\
\hline Days to $50 \%$ flowering & -1.113 & 1.780 & -0.011 & -0.602 & -0.053 & 0.141 & 0.064 & -0.037 & -0.111 & 0.045 & 0.226 & -0.349 & -0.020 \\
\hline Days to maturity & -1.056 & 1.876 & 0.035 & -0.301 & 0.010 & 0.154 & -0.432 & 0.180 & -0.030 & 0.190 & -0.077 & -0.463 & 0.087 \\
\hline Plant height (cm) & 0.104 & 0.576 & 0.114 & -0.045 & -0.062 & -0.586 & 0.056 & -0.169 & 0.005 & 0.078 & 0.103 & -0.529 & $-0.356^{*}$ \\
\hline Number of primary branches & 0.884 & -0.744 & -0.007 & 0.758 & -0.001 & -0.063 & 0.261 & 0.484 & 0.105 & -0.200 & -0.244 & -1.206 & 0.027 \\
\hline Number of mature pods / plant & 0.278 & 0.092 & -0.033 & -0.004 & 0.212 & 1.323 & -0.394 & 0.091 & 0.014 & 0.021 & -0.394 & -0.408 & $0.798 * *$ \\
\hline Pod yield /plant (g) & -0.093 & 0.171 & -0.039 & -0.028 & 0.166 & 1.690 & -0.699 & 0.069 & -0.102 & 0.063 & -0.157 & -0.042 & $0.986^{* *}$ \\
\hline Hundred pod mass (g) & 0.051 & 0.579 & -0.005 & -0.141 & 0.060 & 0.843 & -1.401 & 0.614 & -0.130 & 0.378 & -0.140 & -0.169 & $0.539 * *$ \\
\hline Shelling per cent (S \%) & -0.033 & -0.266 & 0.015 & -0.289 & -0.015 & -0.092 & 0.677 & -1.270 & -0.057 & -0.296 & 0.349 & 0.118 & -0.158 \\
\hline Hundred seed weight (g) & -0.406 & 0.184 & -0.002 & -0.262 & -0.010 & 0.568 & -0.599 & -0.237 & -0.304 & 0.128 & 0.372 & 0.837 & 0.268 \\
\hline Sound mature kernel (SMK \%) & 0.065 & -0.47 & -0.012 & 0.200 & -0.006 & -0.141 & 0.698 & -0.494 & 0.051 & -0.760 & 0.186 & 0.586 & -0.097 \\
\hline Protein content (\%) & -0.231 & 0.516 & 0.036 & 0.543 & 0.051 & 0.042 & -0.140 & 0.843 & 0.151 & 0.264 & -0.378 & -1.684 & 0.013 \\
\hline
\end{tabular}

Residual $=-0.2501$

$*, * *$ - Significant at $5 \%$ and $1 \%$ level of significance, respectively 
Table.6 Genotypic path coefficient analysis showing

\begin{tabular}{|c|c|c|c|c|c|c|c|c|c|c|c|c|c|}
\hline Characters & \begin{tabular}{|c|} 
Days to \\
$50 \%$ \\
flowering
\end{tabular} & $\begin{array}{l}\text { Days to } \\
\text { maturity }\end{array}$ & $\begin{array}{l}\text { Plant } \\
\text { height } \\
\text { (cm) }\end{array}$ & $\begin{array}{c}\text { Number of } \\
\text { primary } \\
\text { branches }\end{array}$ & $\begin{array}{l}\text { Number } \\
\text { of } \\
\text { mature } \\
\text { pods / } \\
\text { plant }\end{array}$ & $\begin{array}{c}\text { Pod } \\
\text { yield/pla } \\
\text { nt (g) }\end{array}$ & $\begin{array}{c}\text { Hundred } \\
\text { pod mass } \\
(\mathrm{g})\end{array}$ & $\begin{array}{l}\text { Shelling } \\
\text { per cent } \\
\text { (S \%) }\end{array}$ & $\begin{array}{l}\text { Hundred } \\
\text { seed } \\
\text { weight (g) }\end{array}$ & $\begin{array}{c}\text { Sound } \\
\text { mature } \\
\text { kernel } \\
\text { (SMK } \\
\%)\end{array}$ & $\begin{array}{c}\text { Oil content } \\
(\%)\end{array}$ & $\begin{array}{c}\text { Protein } \\
\text { content } \\
(\%)\end{array}$ & $\begin{array}{c}\text { Genotypic } \\
\text { correlation } \\
\text { with Kernel } \\
\text { yield/plant } \\
\text { (g) }\end{array}$ \\
\hline & 1 & 2 & 3 & 4 & 5 & 6 & 7 & 8 & 9 & 10 & 11 & 12 & \\
\hline Days to $50 \%$ flowering & -0.211 & 0.237 & -0.031 & -0.001 & -0.014 & 0.282 & -0.028 & 0.048 & -0.054 & 0.003 & 0.034 & -0.076 & 0.189 \\
\hline Days to maturity & -0.169 & 0.294 & 0.047 & 0.000 & -0.002 & 0.090 & -0.056 & 0.113 & -0.007 & -0.011 & -0.006 & -0.215 & 0.077 \\
\hline Plant height (cm) & 0.047 & 0.098 & 0.141 & 0.000 & -0.035 & -0.287 & 0.002 & -0.082 & -0.013 & -0.005 & 0.011 & -0.153 & -0.278 \\
\hline $\begin{array}{l}\text { Number of primary } \\
\text { branches }\end{array}$ & 0.109 & -0.061 & -0.014 & 0.002 & -0.020 & -0.117 & 0.025 & 0.190 & 0.038 & 0.002 & -0.024 & -0.188 & -0.057 \\
\hline $\begin{array}{l}\text { Number of mature pods / } \\
\text { plant }\end{array}$ & 0.023 & -0.005 & -0.039 & 0.000 & 0.126 & 0.899 & -0.053 & 0.036 & 0.011 & -0.001 & -0.054 & -0.145 & $0.796 * *$ \\
\hline Pod yield/plant (g) & -0.052 & 0.023 & -0.035 & 0.000 & 0.098 & 1.152 & -0.097 & 0.026 & -0.065 & -0.004 & -0.020 & -0.034 & $0.993 * *$ \\
\hline Hundred pod mass (g) & -0.031 & 0.086 & -0.002 & 0.000 & 0.035 & 0.580 & -0.192 & 0.244 & -0.081 & -0.021 & -0.019 & -0.067 & $0.533 * *$ \\
\hline Shelling per cent (S \%) & 0.020 & -0.065 & 0.023 & -0.001 & -0.009 & -0.059 & 0.091 & -0.511 & -0.044 & 0.016 & 0.047 & 0.292 & -0.199 \\
\hline Hundred seed weight (g) & -0.058 & 0.011 & 0.009 & 0.000 & -0.007 & 0.383 & -0.080 & -0.114 & -0.195 & -0.007 & 0.053 & 0.248 & 0.243 \\
\hline $\begin{array}{l}\text { Sound mature kernel } \\
\text { (SMK \%) }\end{array}$ & -0.014 & -0.076 & -0.017 & 0.000 & -0.004 & -0.107 & 0.095 & -0.198 & 0.034 & 0.043 & 0.019 & 0.138 & -0.088 \\
\hline Oil content $(\%)$ & -0.067 & -0.016 & 0.015 & 0.000 & -0.064 & -0.219 & 0.034 & -0.227 & -0.098 & 0.008 & 0.106 & 0.227 & $-0.302 *$ \\
\hline Protein content $(\%)$ & -0.032 & 0.126 & 0.043 & 0.001 & 0.036 & 0.077 & -0.025 & 0.297 & 0.096 & -0.012 & -0.048 & -0.502 & 0.058 \\
\hline
\end{tabular}

Residual $=-0.0736$

$*$, ** -Significant at 5\% and $1 \%$ level of significance, respectively 
High genetic advance as percentage of mean was observed for plant height, length of primary branch, number of primary branches, number of mature pods/plant, pod yield/plant, kernel yield/plant, number of one seeded pods $\%$, number of three seeded pod \%, pod length, pod width, hundred pod mass, shelling $\%$, seed length, seed width, hundred seed weight and SMK \%. It indicated that the characters were controlled by additive gene action and selection would be effective for improvement of these characters in genotypes studied. It indicated the necessity of utilising these traits for crop improvement for groundnut. Similar findings of high genetic advance as per cent of the mean for primary branches per plant, kernel yield and pod yield were also reported by Hampannavar et al., (2018).

In the present study high heritability coupled with low genetic advance was observed for oil content and protein content in both the seasons suggesting that variability in this character was due to non additive gene action. In the present investigation, number of mature pods/plant, pod yield/plant and hundred pods mass, showed high positive association with kernel yield, thus suggesting that these characters would be important yield components and the effective improvement in yield could be achieved through selection based on these characters.

From the path analysis study, it was observed that, during kharif season days to maturity had the highest and positive direct effect on kernel yield per plant followed by pod yield/plant, oil content, number of primary branches and plant height, while during summer, pod yield/plant had the highest and positive direct effect on kernel yield per plant followed by days to maturity, plant height, number of mature pods/plant, oil content, SMK\% and number of primary branches. Characters such as number of mature pods/plant, pod yield/plant, showed positive and significant genotypic correlation with kernel yield, exhibiting positive direct effects also. Therefore, selection for these component traits may increase pod yield in studied groundnut genotypes. Similar trend was also observed by Tirkey et al., (2018) for kernel yield and by Zaman et al., (2011) for kernel yield.

In conclusions the both the seasons, most of the characters exhibited high GCV, PCV, heritability and genetic advance per cent over mean. Most of the yield attributing characters showed direct and positive effect on kernel yield.

\section{References}

Allard, R.W. (1960), Principles of plant breeding, John Wiley and Sons, Inc., New York.

Burton, G. W. (1952). Quantitative inheritance in grasses. Proceedings of 6th International Grassland Congress 1.pp.227-83.

Chavadhari, R. M., Kachhadia, V. H., Vachhani, J. H. and Virani, M. B. (2017). Genetic variability studies in groundnut (Arachis hypogaea L.). Electron. J. Plant Breed., 8(4): 12881292.

Dewey, D. R. and Lu, K. H. (1959). A correlation and path coefficient analysis of components of creasted wheat grass seed production Agron. J., 51: 515-18. DOI: http://dx.doi.org/10.1111/j.14390523.2011.01911.x

Fisher, R. A. and Yates, F. (1963). Statistical Tables for Biological, Agricultural and Mestical Res., Oliver and Boyd, Endinburgj.

Gupta, R., Vachhani, J. H., Kachhadia, V. H., Vaddoria, M. A. and Reddy, P. (2015). Genetic variability and 
heritability studies in Virginia groundnut (Arachis hypogaea L.). Electron. J. Plant Breed., 6(1):253256.

Hampannavar, M. R., Khan, H., Temburne, B. V., Janila, $\mathrm{P}$ and Amaregouda, A. (2018). Genetic variability, correlation and path analysis studies for yield and yield attributes in groundnut (Arachis hypogaea L.). J. Pharmacogn. Phytochem., 7(1): 870-874

Hazel, L. N.; Baker, M. L. and Reinmillex, C. F. (1943). Genetic and environmental correlation between the growth rates of pigs at different ages. J. Ani. Sci., 2: $118-28$

Johanson, H.W., Robinson, H.F. and Comstock, H.F. (1955) Estimates of genetic and environmental variability in soybean. Agron. J., 47: 314-18.

Lush, J. L. (1940). Inter size correlation and regression of offspring on dams as a method of estimating heritability of characters. Proc. American Soc. Animal Prod., 33: 293- 301.

Patil, S. K., Shivanna, S., Irappa B. M. and Shweta. (2015). Genetic variability and character association studies for yield and yield attributing components in groundnut (Arachis hypogeae L.). International Journal of Recent Scientific Research, 6(6): 4568-4570.

Tirkey, S. K., Ahmad, E. and Mahto, C. S. (2018). Genetic variability and character association for yield and related attributes in groundnut (Arachis hypogaea L.). Journal of Pharmacogn. Phytochem., SP1: 24872489.

Wright, S. (1921). Correlation and causation. J. Agric. Res. 20: 257-87.

Yusuf, Z., Zeleke, H., Mohammed, W., Hussein, S. and Hugo, A. (2017). Estimate of Genetic Variability Parameters among groundnut (Arachis hypogaea L.) genotypes in Ethiopia. Int. J. Plant Breed. Crop Sci., 4(2): 225-230.

Zaman, M. A., Tuhina-Khatun M., Ullah, M. Z., Moniruzzamn, M. and Alam, K. H. (2011). Genetic Variability and Path Analysis of Groundnut (Arachis hypogaea L.). The Agriculturists, 9(1\&2):29-36.

\section{How to cite this article:}

Kalyani Kumari and Sasidharan, N. 2020. Studies on Genetic Variability, Correlation and Path Coefficient Analysis for Morphological and Yield Traits in Different Arachis spp. Int.J.Curr.Microbiol.App.Sci. 9(11): 1030-1039. doi: https://doi.org/10.20546/ijcmas.2020.911.121 